\title{
1st Grade Completion
}

National Cancer Institute

\section{Source}

National Cancer Institute. 1st Grade Completion. NCI Thesaurus. Code C67123.

Indicates that 1 st grade is the highest level of educational achievement. 